dieselben elektrolytisch, mit Hilfe des Platinelektrolysators herzustellen.

Sobald ein geeigneter Platz zur Anlage gewählt wird, dürfte es auf diesem. Wege gelingen, das Chlor zo einem relativ niederen Kostenpreise zu gewinnen. Damit wäre aber auch gleichzeitig die Möglichkeit geschaffen, die Gestehungskosten der daraus erhältlichen Produkte und Präparate entsprechend zu ermäßigen.

\section{Ein Fortschritt auf dem Gebiete der indirekten Farbenphotographie.}

\author{
(Jan Szczepaniks neuer Mehrfarbenraster.) \\ Mitteilung von $F_{R}$. Limmfr. \\ (Eingeg. d. 16./12. 1908.)
}

Die L u m i è $r$ esohe Autochromplatte ist auf dem Gebiete der indirekten Farbenphotographie ein großer, äußerst dankenswerter Fortschritt gewesen. Ein Fortschritt, den man erst ganz zu würdigen versteht, wenn man selbst auf ähnlichem Gebiete arbeitet, wenn man aus eigener Erfabrung weiß, wieviel Ausdauer und wieviel Kleinarbeit gerade auf farbenphotographischem Gebiete für jede Vervollkommnung nötig sind.

Die Autochromplatte hat sich in kurzer Zeit die Welt erobert. Während nun Fachwelt und Amateure sich dieser neuesten Errungenschaft der Farbenphotographie herzlich freuen, hat Herr J a $\mathbf{n}$ $\mathrm{Sz}$ z z e p a $\mathrm{n}$ i $\mathrm{k}$ (der den Herren Fachgenossen als Erfinder des ,dreischichtigen Ausbleichpapiers" bekannt sein wird) bereits am 14./8. 1907 ein für die indirekte Farbenphotographie wichtiges Verfahren zum Patent angemeldet. Ein Verfahren, das, wenn es die Vorteile hält, welche es verspricht, dem $\mathrm{L} u \mathrm{~m}$ i è $\mathrm{r}$ eschen Verfahren wohl Konkurrenz machen wird.

Ich will zunächst mit ein paar kurzen Worten auf die Autochromplatte eingohen. (Mit ,Autochromplatte" wird eine von $\mathbf{L} \mathbf{u ~} \mathrm{m}$ i è $\mathrm{re}$ in Lyon fabrizierte photographische Platte bezeichnet, welche es ermöglicht, mit Hilfe einer einzigen Aufnahme ein annähernd farbenrichtiges Bild eines farbigen Gegenstandes herzustellen.) Der wesentlichste Teil der Autochromplatte ist ein auf sehr geniale Weise verfertigter Dreifarbenkornraster. Dieser Raster wird hergestellt aus gefärbten Stärkekörnchen. Auf eine geeignet präparierte Platte werden blau, grün und rot gefärbte Stärkekörnohen (in einem bestimmten Verhältnis gemischt) aufgestäubt. Die unvermeidlichen kleinen Räume zwischen den einzelnen Stärkekörnchen werden mit einer schwarzen Masse ausgefüllt. Die Lichtempfindlichkeit der Autochromplatte ist eine verhältnismäBig geringe. Dies hat, seine Ursache zum Teil in der ungenügenden Lichtdur chlässigkeit der Stärkekörnchen, die schwarze Füllmasse hält natürlich auch Licht zurück. Diese beiden Fehler weist der $\mathbf{S}$ z c z e p a n i k sche Dreifarbenraster nicht auf. Ich will nur das Wichtigste aus der sehr umfangreichen Patentschrift mitteilen.

$\mathrm{Sz}$ czepanik stützt sich bei seinem neuen Verfahren auf das Gesetz der „Farbenwanderung". Dieses Gesetz der Farbenwanderung ist aus Erfahrung gewonnen, eine einleuchtende Erklärung dafïr ist mir bis jetzt nicht bekannt geworden. Es haben gewisse Farbstoffe eine Vorliebe für gewisse Farbstoffträger. Gibt man z. B. auf eine farblose Gelatineschicht eine mit Erythrosin (rot) gefärbte Kollodiumschicht, so "wandert" das Erythrosin (fast) vollständig aus dem Kollodium in die Gelatine. Man hat (mit wenigen Ausnahmen!) die Tatsache feststellen können, daß basische Farbstoffe eine ausgesprochene Zuneigung zu Kollodium, saure Farbstoffe eine große Vorliebe für Gelatine besitzen. Diesen Neigungen entspricht auch das „Wanderungsbedürfnis", d. h. sobald 'Gelegenheit gegeben ist, ,wandern" die sauren Farbstoffe, von Kollodium in Gelatine und die basischen von Gelatine in Kollodium. Es gibt nun Farbstoffträger, welche sich den Farbstoffen gegenüber ähnlich wie Kollodium und solche, die sich annähernd wie Gelatine verhalten. Gummipulver z. B. zeigt ähnliche Eigenschaften wie Gelatine.

$\mathrm{S} z$ c ze p a $\mathrm{n}$ ik stellt sich drei Lösungen aus Gelatine oder Gummi her. Diese werden mit drei verschiedenen passenden Farbstoffen angefärbt und die Lösungen dann vorsichtig ,,zur Trockne" eingedampft. Die betreffenden Farbstoffe müssen natürlich eine ,Zuneigung für" Kollodium haben, technisch ausgedrückt ,kollodiumfreundlich" sein. Die durch das Eintrocknen gewonnenen "festen “ Gelatine- oder Gummimassen werden fein pulverisiert und die drei verschiedenfarbigen. Pulver sorgfältigst gemischt. Die Farbpulvermischung wird mittels geeigneter Einstaubapparate auf eine noch etwas feuchte Kollodiumplatte gesiebt. Die Farbstoffe wandern aus dem Gelatinepulver in die Kollodiumfläche und es entsteht ein Mosaik von kleinen, bunten Feldern, ähnlich dem Stärkekörnchenraster der Autochromplatte. Das aufgestäubte, farbarm gewordene Pulver wird weggewaschen.

Es kommt allerdings vor, daß beim Aufstäuben die einzelnen Farbpulverteilchen sich überdecken. Ein Farbenaustausch der Teilchen untereinander tritt trotzdem nicht ein. Die überdeckenden Teilehen werden gleichzeitig mit den farbarmen Teilchen weggewaschen. Infolge ungenügenden Kontaktes zwischen Pulver und Kollodiumschicht entstehen an manchen Stellen farblose Felder. Dieser Fehler läßt sich u. a. dadurch ausgleichen, daß man beim Einstauben von einem der Farbpulver etwas weniger anwendet, als an sich nötig ist. Die eingestaubte Platte wird dann in einer Gelatinefarblösung desjenigen Farbstoffes gebadet, von welchem weniger zum Einstauben gewonnen wurde. Auf diese Weise werden auch die farblosen Felder in zweckentsprechender Weise angefärbt.

Man erhält nach dem S z c z e p a n i k schen Verfahren einen Dreifarbenraster, der eine Reihe offenkundiger Vorzüge vor dem Raster der Autochromplatte besitzt.

Der Szczepaniksche Raster ist wesentlich lichtdurchlässiger als der Autochromraster. Er besitzt keinerlei Füllmasse. Ein Farbfeld liegt direkt am andern. Die Vorteile, die sich daraus ergeben, sind: ,Eine größere Lichtempfindlichkeit der Platte. Eine erhöhte Brillanz und vermehrte Helligkeit der Bilder. Ferner Parallaxenfreiheit, was für das ev. Kopieren der Platten sehr wesentlich ist." 
Die neuen Platten sind vorläufig noch nicht im Handel zu haben. Wenn sie kommen und das halten, was der Frfinder hofft, so bedeutet das $\mathrm{Sz}$ e z e pan $\mathrm{ik}$ sche Verfahren wirklich einen weiteren Fortschritt auf dem Gebiete der indirekten Farbenphotographie. Szczepaniks sinnreiche An. wendung des „Farbenwanderungsgesetzes" dürfte für eine ganze Anzahl von Industriezweigen eine praktische Bedeutung gewinnen.

K u $1 \mathrm{~m} \mathrm{ba} \mathrm{ch}$, am 9. Dez. 1908.

\section{Besprechung neuer Farbstoffe und Musterkarten für Färberei und Druckerei.}

\author{
Von P. Krars, Tübingen.
}

(Eingeg. d. 18./12. 1908.)

Bis zum 15. Dezember 1908 ist folgendes eingegangen (vgl. 1908 21, S. 686, 1073, 1745 und 1988.)

\section{Badische Anilin- und Soda-Fabrik.}

Substantive Farbstoffe a uf B a $\mathbf{u} \mathbf{m}$ oll garn. Ein prachtvoll ausgestattetes Musterbuch, in dem an 432 Mustern die mit 5 verschiedenen Färbeverfahren herstellbaren Fär. bungen mit den Baumwollfarbstoffen dieser Firma illustriert werden.

Kryogendirektblau 3 B und 3 B e x tr a. Prospekt und je eine Musterkarte für lose Baumwolle und für Garn. Es werden lebhafte Dunkelblaus erzielt.

Stilbengelb G. Prospekt und Musterkarte für Baumwollgarn. Ein reines Goldgelb von guten Echtheitseigenschaften.

F u s c a m in G. Prospekt und Musterkarte. Es ist dies ein Braun für Baumwolldruck, das sich mit Rongalit $C$ weißätzen läßt.

M a rine b l a u RR, R und BBT f ü r W o l l d r u c k. Prospekt und Musterkarte. Die Lichtechtheit und die Leichtigkeit, mit der selbst dunkle Töne gleichmäßig ausfallen, sind hervorgehoben.

\section{Leopold Cassella co.}

Prospekt und Musterkarte von Diamina z o s charlach $4 \mathrm{~B}$ und $8 \mathrm{~B}$, zwei neue Entwicklungsfarbstoffe, die sich für alle $Z$ weige der Baumwollfärberei eignen und durch gute Echtheit auszeichnen.

D i a m in oge n b la u $6 \mathrm{RN}$ gehört zu der lichtechten Diaminogenblaugruppe und ist etwas röter bzw. violetter als die älteren Marken.

Diaminbrillantviolett $R R$ und $B$, zwei neue Direktvioletts von besonders lebhafter und klarer Nuance.

\section{Farbenfabriken vorm. Fr. Bayer \& Co.} Wollf ärberei.

Monochromfarben auf Kamm g a r n. Eine Musterkarte enthaltend 72 Färbungen, die so hergestellt sind, daB Farbstoff und Bichromat im gleichen Bad zusammen zur Verwendung kommen, wodurch das Musterfärben sehr erleichtert wird.
Moderne Herrenst offe 1908-1909. Acht buntfarbige Herrenstoffmuster nebst den sie zusammensetzenden Garnfärbungen.

Sulfoncyanins chwarz $4 \mathrm{~B}$ und BR. Zwei neue Nuancen, die sich den älteren Marken anschließen und diesen in Echtheit usw. gleichstehen.

S u lf on s ä u regrün B. Ein dunkles Blaugrün, dessen Echtheit und gute Färbeeigenschaften hervorgehoben werden.

Vict oria marine bla u LH. Diese neue Marke besitzt wesentlich bessere Lichtechtheit als die ältere Marke DK.

Brillantsäurebla u FF. Ein leuchtend klares egalisierendes Wollblau.

T u c hrot BC, gibt lebhafte volle Bordeauxtöne von guter Echtheit.

$$
\text { B a u m w llf ärberei. }
$$

Katigentiefsch w a $\mathrm{z}$ R. Eine neue Marke, die besonders volle schöne Schwarz gibt und sehr ausgiebig ist.

Diazoechtschwarz MG gibt beim Entwickeln mit Entwickler A ein volles Blauschwarz, mit Entwickler $H$ ein gedecktes Tiefschwarz.

B en z o e c h t s c har la ch 7 BS. Ein säureechtes und gut lichtechtes lebhaftes Rot.

A l g o lr ot $5 \mathrm{G}$ in Teig. Dieser neue Küpenfarbstoff liefert klare, gelbstichige Rosa bis Ziegelrot von der hervorragenden Echtheit der Farbstoffe dieser Klasse.

$$
\text { Leinenf är berei. }
$$

Katigensohwarzauf Leinennäh. g a $r$ n. Eine Kollektion von 12 Musterrollen, die mit den verschiedenen Katigenschwarzmarken gefärbt sind.

\section{Druckere $i$}

P a $\mathbf{r}$ a $\mathrm{s}$ c h w a r z R. Ein Kupplungsfarbstoff für Ätzdruck. Auf dèn Jigger vorfärben, mit diazotiertem Nitranilin entwickeln. Man erhält braunstichige Schwarznuancen, die mit Rongalit $\mathrm{C}$ rein weiß ätzbar sind.

Parabraun $R$, wird ebenso behandelt.

Druckverfahren für Katigen f a $r$ b t of $f e$. Dieses neue Druckverfahren, das durch 12 sehr schöne Muster in verschiedenen Farben illustriert wird, beruht darauf, daß man statt der bisher zur Verdickung gebrauchten Dextrinoder Britishgum-Mischungen (die, wie sich herausgestellt hat, die Ausgiebigkeit der Farbstoffe sehr beeinträchtigen) mit Natronlauge aufgeschlossene Stärke, insbesondere Maisstärke verwendet. Gleichzeitig mit diesem Rezept bringt die Firma eine Anzahl Katigenfarben in gereinigter hochkonzentrierter Pulverform in den Handel.

\section{Farbwerke vorm. Meister Lueius Brüning.}

Wollf ärberei.

Einbadige walk- und tragechte Braunnuancen a $\mathbf{n}$ Wolle und Kammg a r n. 12 Färbungen, die mit Alizarinfarben durch Nachchromieren im gleichen Bad hergestellt sind.

Sä urealizarinbraun RR, ein neuer Chromentwicklungsfarbstoff von sehr reiner rotbrauner Nuance. 